\title{
Painful and Painless Diabetic Neuropathies: What Is the Difference?
}

\author{
Pallai Shillo ${ }^{1}$. Gordon Sloan ${ }^{1} \cdot$ Marni Greig ${ }^{1} \cdot$ Leanne Hunt $^{1} \cdot$ Dinesh Selvarajah ${ }^{2} \cdot$ Jackie Elliott $^{2} \cdot$ Rajiv Gandhi $^{1}$. \\ lain D. Wilkinson ${ }^{3} \cdot$ Solomon Tesfaye ${ }^{1,2}$
}

Published online: 7 May 2019

(C) The Author(s) 2019

\begin{abstract}
Purpose of Review The prevalence of diabetes mellitus and its chronic complications are increasing to epidemic proportions. This will unfortunately result in massive increases in diabetic distal symmetrical polyneuropathy (DPN) and its troublesome sequelae, including disabling neuropathic pain (painful-DPN), which affects around $25 \%$ of patients with diabetes. Why these patients develop neuropathic pain, while others with a similar degree of neuropathy do not, is not clearly understood. This review will look at recent advances that may shed some light on the differences between painful and painless-DPN.

Recent Findings Gender, clinical pain phenotyping, serum biomarkers, brain imaging, genetics, and skin biopsy findings have been reported to differentiate painful- from painless-DPN.

Summary Painful-DPN seems to be associated with female gender and small fiber dysfunction. Moreover, recent brain imaging studies have found neuropathic pain signatures within the central nervous system; however, whether this is the cause or effect of the pain is yet to be determined. Further research is urgently required to develop our understanding of the pathogenesis of pain in DPN in order to develop new and effective mechanistic treatments for painful-DPN.
\end{abstract}

Keywords Diabetes $\cdot$ Peripheral neuropathy $\cdot$ Neuropathic pain $\cdot$ Small fiber neuropathy $\cdot$ Painful diabetic neuropathy $\cdot$ Diabetic neuropathy

\section{Introduction}

The worldwide prevalence of diabetes mellitus (DM) has reached epidemic proportions, and is set to increase to 629

Pallai Shillo and Gordon Sloan are joint first authors

This article is part of the Topical Collection on Microvascular Complications-Neuropathy

Solomon Tesfaye

solomon.tesfaye@sth.nhs.uk; s.tesfaye@sheffiedl.ac.uk

Pallai Shillo

shillopr@yahoo.com

Gordon Sloan

Gordon.sloan@nhs.net

Marni Greig

Marni.Greig@sth.nhs.uk

Leanne Hunt

Leanne.Hunt@sth.nhs.uk

Dinesh Selvarajah

Dinesh.Selvarajah@sth.nhs.uk million by 2045 [1]. Rising population growth, aging, urbanization, and an increased prevalence of obesity and physical inactivity are amongst the major contributing factors. Diabetic neuropathies are one of the most common chronic
Jackie Elliott

Jackie.Elliott@sth.nhs.uk

Rajiv Gandhi

Rajiv.Gandhi@sth.nhs.uk

Iain D. Wilkinson

i.d.wilkinson@sheffield.ac.uk

Diabetes Research Unit, Royal Hallamshire Hospital, Sheffield Teaching Hospitals NHS Foundation Trust, Glossop Road, Sheffield S10 2JF, UK

2 Department of Oncology and Human Metabolism, University of Sheffield, Sheffield, UK

3 Academic Unit of Radiology, University of Sheffield, Sheffield, UK 
complications of DM [2], and distal symmetrical polyneuropathy (DPN) is the most prevalent form of diabetic neuropathy, which may affect up to $50 \%$ of patients $[2,3 \cdot 4 \cdot]$. The Toronto Expert Group has defined DPN as "a symmetrical, length dependent sensorimotor polyneuropathy attributable to metabolic and micro-vessel alterations as a result of chronic hyperglycaemia exposure and cardiovascular risk covariates" [5•]. A more recent definition of DPN in the American Diabetes Association Position Statement is "the presence of symptoms and/or signs of peripheral nerve dysfunction in people with diabetes after the exclusion of other causes" [3•]. The rising numbers of patients diagnosed with neuropathic disorders related to DM will have an immense impact on health and social care provision [6].

DPN is a major risk factor for diabetic foot ulceration, which remains a major cause of morbidity and is the leading cause of non-traumatic amputations [7]. Although a large number of patients with DPN may be entirely asymptomatic, approximately $15-25 \%$ of people with DM present with neuropathic pain (painful-DPN) $[8-11,12 \cdot, 13]$. The neuropathic pain is of varying degree of intensity [14] DPN and painfulDPN has different clinical syndromes with the most common of which is a mixed large and small fiber neuropathy. Small nerve-fibers (SF) are small-caliber sensory fibers, which are primarily responsible for peripheral nociception [15]. Pure SF neuropathy may occur in DM and the clinical features include symptoms of painful peripheral neuropathy with signs of SF impairment (e.g., pinprick or thermal hypoalgesia or allodynia) in a peripheral neuropathy distribution in the absence of large fiber impairment (e.g., impaired light touch, vibration, proprioception or motor signs).

Painful-DPN often results in insomnia, mood disorders, and a poor quality of life [12•]. The currently available therapies for the pain associated with DPN remain inadequate, given relatively modest pain relief and often troublesome side effects $[3 \cdot 16,17]$. There is thus an urgent need to have a better understanding of the pathogenesis of pain in DPN and this has been the subject of a recent review (Fig. 1) [17]. Central to this understanding will be to develop new insights as to why some patients develop disabling neuropathic symptoms while others with a similar degree of neuropathy do not. This review will discuss the differences in risk factors, clinical features, serum biomarkers, vascular alterations, quantitative sensory testing (QST), skin biopsy parameters, genetics, and brain imaging studies between painful- and painless-DPN.

\section{Risk Factors}

Several risk factors for DPN in general have been described and confirmed in cohorts of type 1 and type 2 diabetes. The EURODIAB Prospective Complications Study screened 3250 type $1 \mathrm{DM}$ patients at baseline and followed 1172 patients without DPN looking for risk factors that predicted the development of DPN [4•]. The study found that in addition to glycemic control, traditional vascular risk factors such as hypertension, raised triglycerides, obesity, and cigarette smoking were independent risk factors for the development of new onset DPN. Similar vascular risk factors were also found in T2DM $[18 \cdot, 19,20]$. However, the risk factors for neuropathic pain in DM are less well known. This is partly because of the wide variation in the diagnostic and population selection methods employed by the epidemiological studies for painful-DPN $[21 \bullet \bullet, 22]$. The reported risk factors include increasing age [9, 10], elevated HbA1c [23••, 24], duration of DM [9], and obesity $[10,25]$. A high alcohol intake, type of diabetes, macro and microvascular disease, and ethnicity have also been implicated $[21 \bullet \cdot]$. Recent large studies have also suggested nephropathy and female gender as risk factors for painful-DPN [26, 27••, $280^{\circ}$. Indeed, female gender was the only risk factor identified in a large cross-sectional study $(n=816)$ performed by Truini et al. which diagnosed painful-DPN using widely agreed criteria [28••]. Thirteen percent were diagnosed with painfulDPN and the only distinguishing risk factor from painless-DPN was female gender. Gender differences are well recognized in chronic pain conditions and neuropathic pain intensity has previously been reported to be more severe in females $[29,30]$.

Recent advances in gene sequencing technology have led to several studies examining genetic variants associated with DPN and painful-DPN [31-34, 35•, 36]. Two recent studies by Meng et al. conducted genome-wide association studies in Tayside, Scotland [32, 33]. Chr8p21.3, Chr1p35.1, and Chr8p21.3 polymorphisms were associated with neuropathic pain. However, the study did not use validated diagnostic criteria for painful-DPN. Recently also, there has been great interest in the role of voltage-gated sodium channels and their role in neuropathic pain. The $\mathrm{Na}_{\mathrm{v}} 1.7$ sodium channel is well recognized to be involved in pain signaling and "gain of function" mutations of its encoding gene, SCN9A, cause rare pain disorders. Additionally, studies have identified $\mathrm{Na}_{\mathrm{v}} 1.7$ mutations in idiopathic small fiber neuropathy [36] and painfulDPN [34]. Blesneac et al. looked at the relationship between $\mathrm{Na}_{\mathrm{v}} 1.7$ variants and painful-DPN and found that none of the participants with painless-DPN $(n=78)$ were found to have a genetic variant [35•]. However, a total of 12 rare $\mathrm{Na}_{\mathrm{v}} 1.7$ variants were identified in 10 out of 111 patients with painful-DPN. The subjects with these variants were found to have a shorter duration of diabetes yet more severe burning pain. Painful-DPN is a heterogeneous condition and subjects with rare sodium channel gene variants may represent a subgroup that may respond to a particular treatment.

In summary, while the risk factors for DPN are well recognized, those for painful-DPN are less certain. This might indicate the complexity of painful-DPN as many factors including genetics, cultural, psycho-social, and gender may be involved. 


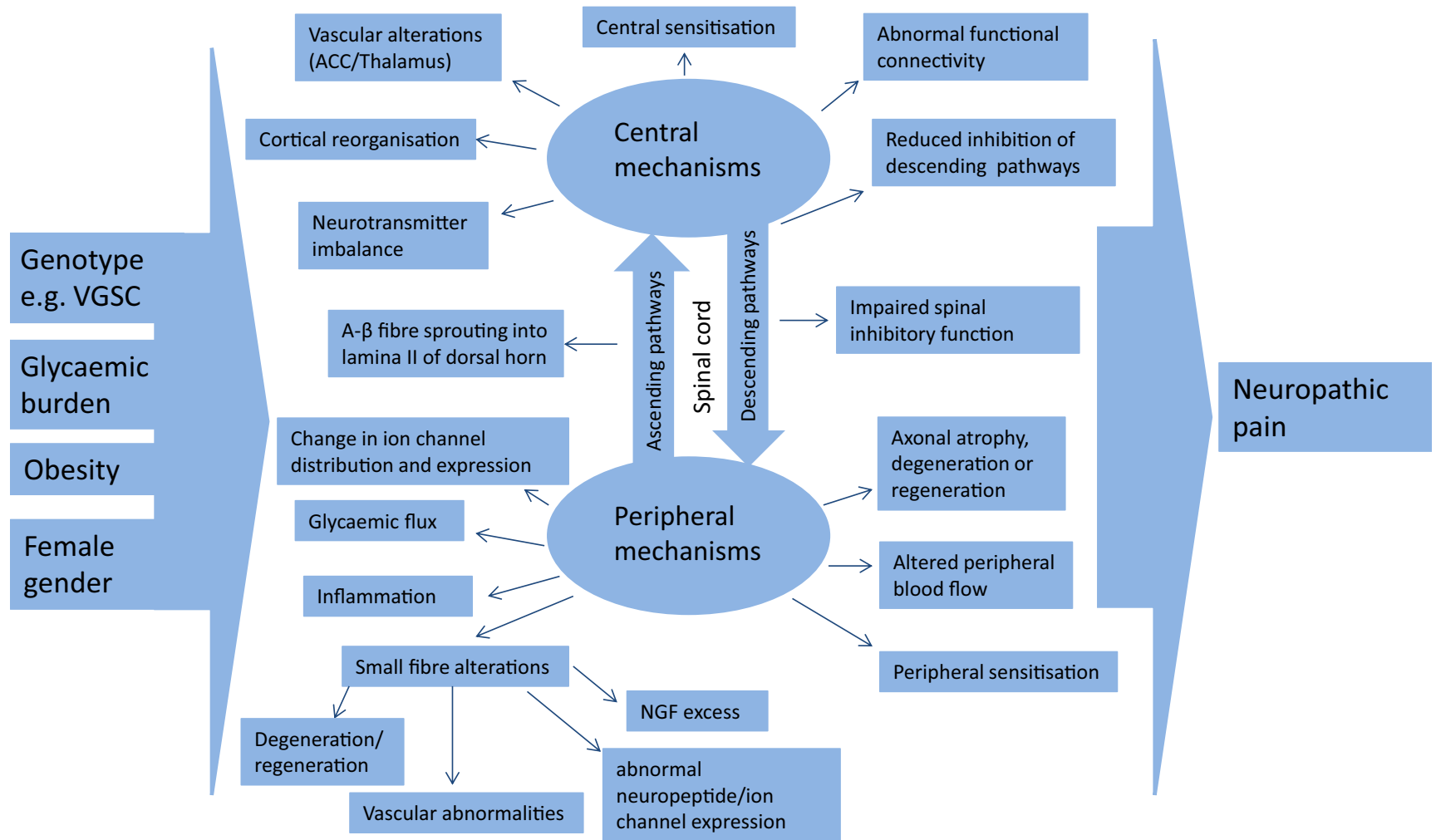

Fig. 1 An overview of the current postulated pathogenesis of painfulDSPN. The risk factors for the generation of neuropathic pain in DSPN may include glycemic burden (duration of diabetes), obesity, female gender, and genetic variants of voltage-gated sodium channels (VGSC).

\section{Clinical Features}

Neuropathic pain in diabetes has distinct presentations as burning, sharp, aching, electric, and evoked pains [37]. However, patients may also describe symptoms of numbness, tingling, and pins and needles, irrespective of the presence of pain. Neuropathic pain may also induce various degrees of physical disability, depression, anxiety, insomnia, and a poorer quality of life than patients with painless-DPN, particularly with moderate to severe neuropathic pain $[23 \bullet \bullet, 27 \bullet \bullet$, 38]. Despite these profound differences in a patient's clinical presentation, there are few distinct differences in the neurological examination between painless- and painful-DPN. The majority of patients with painful-DPN demonstrate sensory loss on clinical examination but a small proportion of patients with painful-DPN have evidence of "gain of function" signs such as allodynia and hyperalgesia [23••]. There is controversy regarding whether the severity of neuropathic impairment is greater in painful-DPN. Several studies have reported a correlation between neuropathy severity and the presence and/or severity of neuropathic pain in DPN $[8,11,15,23 \bullet$, $39-41]$ whereas other studies have not $[18 \bullet, 28 \bullet \bullet$. Although
Both the central and peripheral mechanisms have been postulated in the pathogenesis of painful-DSPN. ACC, anterior cingulate cortex. (Adapted from: Sloan G, et al. Diabetes Res Clin Pract. 2018; 144: 177-91, with permission from Elsevier) [17]

the weight of evidence seems to suggest that an increasing severity of DPN may increase the risk of developing painful neuropathic symptoms, severe DPN and pain are not mutually exclusive, and there may have been a selection bias in recruiting painful-DPN patients from tertiary referral centers.

\section{Cardiovascular Autonomic Neuropathy}

Both autonomic neuropathy and painful-DPN involve small fibers, and a potential relationship was therefore investigated. In a small study, we demonstrated greater changes in heart rate variability studies, as measures of cardiovascular autonomic neuropathy $(\mathrm{CAN})$ in subjects with painful- compared with painless-DPN [42], while other small studies reported that painful DPN was more likely to be associated with the absence of a nocturnal fall in blood pressure ("non-dipping") [43], or with reduced Valsalva ratio [40]. However, these are in contrast with other studies that have not found any differences in measures of CAN between painful and painless-DPN $[39,44,45]$. 


\section{Diagnostic Methods of Painful-DPN}

Conventional neurophysiological testing methods, which measure large fiber function, such as nerve conduction studies (NCS), cannot detect pure small fiber neuropathy (SFN) [46]. However, QST and more recent advances in diagnostic techniques, e.g., skin biopsy with intraepidermal nerve fiber density (IENFD) quantitation, corneal confocal microscopy (CCM), and laser Doppler imaging flare (LDI Flare) have allowed the reliable diagnosis of SFN $[5 \bullet, 46]$. Because of their role in physiological nociception, studies have explored whether damage or alterations in SF may relate to neuropathic pain in DPN.

\section{Skin Biopsy}

Immunostaining of skin biopsy samples with protein gene product 9.5 and quantitation of IENFD is a reliable means of diagnosing SFN [46]. However, IENFD is unable to distinguish between individuals with or without neuropathic pain [23・•, $27 \bullet \bullet, 28 \bullet \bullet, 47 \bullet \bullet, 48,49$ ]. Other studies have been performed to determine whether morphological and functional markers of the epidermal innervation revealed differentiating features. Intraepidermal nerve fiber (IENF) regeneration, by measuring the ratio of growth associated protein-43 (GAP-43) to nerve fibers, has been shown to be enhanced in painful-compared with painless-DPN $[49,50 \bullet, 51]$. However, Scheytt et al. found no relationship between pain and GAP-43 reactivity in subjects with peripheral neuropathies of varying etiologies [52]. There are contradictory findings in studies investigating other IENF markers to differentiate painful- from painless-DPN including IENF length $[50 \bullet, 53]$ and axonal swellings, which are measures of axonal degeneration [49,54]. Levels within the skin of the neurotrophin nerve growth factor (NGF) were increased in patients with DPN and sensory symptoms, including pain, compared to painless-DPN [55]. NGF has recently been shown to sensitize nociceptors in human skin and it has been hypothesized that the remaining IENF in painful-DPN may be exposed to excessive levels of NGF ("over-trophing") resulting in hypersensitivity and neuropathic pain [19, 55-58].

\section{Corneal Confocal Microscopy}

Confocal corneal microscopy (CCM) can rapidly, noninvasively, and accurately image corneal nerves and is a recently developed diagnostic test for DPN [59-61]. Studies of $\mathrm{CCM}$ have explored the role of corneal innervation and neuropathic pain in DM $[53,62 \cdot, 63]$. Quattrini et al. reported reduced corneal nerve fiber length with unaltered other CCM measures [53], whereas Marshall et al. found unaltered corneal nerve fiber length but reduced corneal nerve fiber density [62•]. Recently, Kalteniece et al. [63] described significantly lower corneal inferior whorl length, and average and total nerve length in painful- compared to painless-DPN. Changes within this region have been suggested to be indicative of early neuropathic damage. However, there were confounding factors, which could account for these group differences. Therefore, the association of CCM abnormalities to neuropathic pain in DPN is thus far inconclusive.

\section{Evoked Responses}

Non-invasive tests have been developed to investigate the peripheral function of SF to diagnose SFN. Such tests can measure evoked potentials in response to stimuli that activate the nociceptive pathway, for example contact heat-evoked potentials (CHEPS) [64]. CHEPs correlates with other measures of SFN including IENFD and leg skin flare responses $[65,66]$. One small study found a relationship between enhanced brain CHEP amplitudes in subjects with painful-DPN; this result was most marked in those with thermal hyperalgesia and mechanical allodynia [67].

\section{Quantitative Sensory Testing}

QST is a psychophysical measure of the perception of different external stimuli of controlled intensity to assess a range of sensory modalities [68, 69]. Some studies with a relatively small sample size suggested that conventional QST measures of SF function may be statistically different between painfuland painless-DPN [41, 44, 70, 71]. More recent studies have employed the German Research Network on Neuropathic Pain (DFNS) QST protocol to quantify sensory loss, for small and large fiber function, and sensory gain abnormalities [72, 73.•]. Three recent large cross-sectional cohort studies have applied this protocol to patients with painful- and painlesspolyneuropathies with different etiologies [48] and painfuland painless-DPN $[23 \bullet \bullet, 27 \bullet \bullet]$. In two studies of painfulDPN, DFNS QST revealed more severe loss of function in those with neuropathic pain, particularly patients with moderate/severe pain $[23 \bullet \bullet, 27 \bullet \bullet]$. Thermal hyposensitivity was more severe in painful-DPN whereas mechanical stimuli showed fewer differences compared with painless-DPN. Gain of function abnormalities and preserved SF function with hyperalgesia were both rare. However, Üçeyler et al. studied patients with painful- and painless-polyneuropathies of different etiologies and found that patients with neuropathic pain demonstrated elevated mechanical pain and detection threshold, and lower mechanical pain sensitivity with no difference in SF deficits [48]. This perhaps indicates there may be a unique somatosensory phenotype associated with painfulDPN characterized by more severe SF dysfunction with thermal hyposensitivity $[23 \bullet \bullet, 27 \bullet \bullet]$. However, SF changes are common and can occur in early DPN without pain [74-76]; therefore, these findings alone are unable to completely explain why some patients develop neuropathic pain and others 
do not. Perhaps, other investigations into small fiber function and structure, such as skin biopsy studies, may shed further light onto this paradox.

\section{Pathogenesis of Painful-DPN}

\section{Microvascular Blood Flow}

Consistent with vascular risk factors increasing the risk of DPN [4•], both structural and functional microvascular abnormalities of the vasa-nervorum have been shown to be involved in the pathogenesis of DPN [77-79]. Patients with treatment induced neuropathy of diabetes who had extremely severe neuropathic pain have proliferating blood vessels on the epineurial surface bearing striking similarities to those found in proliferative diabetic retinopathy [80]. It is well recognized that very rapid improvement in glucose control can cause proliferative retinopathy mediated by retinal ischemia and a similar process appears to take place in the peripheral nerve. Furthermore, several studies have shown that regulation of peripheral blood flow is altered in patients with painful- compared with painless-DPN [81-84]. Our group demonstrated elevated sural nerve epineurial oxygen saturation and faster blood flow in patients with painful- compared to painlessDPN, perhaps secondary to arteriovenous shunting [82]. Other studies have examined the role of skin microvascular vasodilator and vasoconstrictor responses in subjects with DPN, with contradictory findings [71, 85-87].

Studies measuring serum markers of angiogenesis (vascular endothelial growth factor, VEGF) and endothelial dysfunction (soluble intercellular adhesion molecule - 1, sICAM-1) have found them to be elevated in painful-DPN $[86,88 \cdot]$ and symptomatic DPN respectively [89]. Furthermore, punch skin biopsy studies have also indicated that skin microcirculation may be involved in the pathogenesis of painful-DPN. One study demonstrated evidence of hypoxia, by immunostaining with hypoxia inducible factor $1 \alpha$ (HIF-1 $\alpha$ ), to be related to pain intensity in subjects with DPN [90]. Recently, our group has also found dermal von Willebrand factor (vWF) immunoreactivity, as a blood vessel marker, to be significantly elevated in subjects with painful-DPN, in comparison to subjects with painless-DPN, patients with DM without DPN and healthy volunteers [56]. Moreover, small studies have demonstrated that pain improves with topical application of vasodilator treatments [91, 92], perhaps indicating that local blood flow dysregulation could be a viable target for the management of pain in DPN.

\section{Hyperglycemia and Downstream Effects}

Hyperglycemia mediated metabolic pathways have long been associated in the pathogenesis of DPN, but their role in those with neuropathic pain is less clearly defined. Studies using DM rodent models have found neuropathic pain behaviors to be related to numerous metabolic pathways including the polyol pathway, protein kinase $\mathrm{C}$ activity, and increased advanced glycation end-products (AGEs) [93]. However, there is limited evidence to support glycemic control or lifestyle modifications in improving painful neuropathic symptoms [3•]. Moreover, the evidence to support pathogenic treatments for neuropathic pain in DPN has generally been disappointing and only a few pharmacotherapeutic agents are available in select countries [50•].

Methylglyoxal is a highly reactive dicarbonyl compound and is a precursor to the formation of (AGEs). The formation of AGEs has downstream deleterious effects on peripheral nerves and Schwann cells including inflammation and oxidative stress [94]. Methylglyoxal has been suggested to be an important factor in the development of DM and incident DPN $[20 \bullet, 95]$. In rodent models of painful-DPN, methylglyoxal has been shown to induce hyperalgesia via activation of the voltage-gated sodium channel Nav 1.8 and transient receptor potential channel ankyrin-1 [96, 97]. Similarly, in a small number of patients with DM $(n=30)$, serum methylglyoxal levels were found to be elevated in painful-DPN [96]. In contrast to these findings, a larger study $(n=882)$ reported methylglyoxal levels to be unrelated to painful-DPN [98]. Although the role of hyperglycemia mediated pathways in generating neuropathic pain is uncertain, pathogenically oriented treatments, particularly anti-oxidants, have been demonstrated to improve pain in some pre-clinical and clinical trials $[99,100]$.

\section{Vitamin D}

Although vitamin $\mathrm{D}$ is most commonly recognized for its role in calcium metabolism and bone health, vitamin D is involved in many disparate physiological processes [101]. Deficiency of vitamin D has been shown to be predictive of numerous chronic diseases including DM, DPN, and chronic pain [101-104]. Pre-clinical studies indicate that vitamin D appears to play a critical role in nerve function in health and may play a role in neuropathic pain syndromes [105-109]. Our group recently found vitamin D levels to be significantly lower in patients with painful- compared to painless-DPN, with a significant correlation between serum 25-hydroxyvitamin D level and pain scores on the Doleur Neuropathique 4 neuropathic pain screening tool [110•]. The study was cross-sectional, and therefore cannot establish a causal relationship, but it does suggest a possible mechanistic link between vitamin D and painful-DPN. Indeed, three non-randomized clinical trials have demonstrated an improvement in painful neuropathic symptoms with vitamin D therapy but, further, larger, adequately powered RCTs are necessary to investigate this further [111-113]. 


\section{Inflammation}

Inflammation has been postulated to play a major part in DM and DPN [114]. Low grade inflammation has been suggested as a link between obesity and T2DM, via inflammation induced insulin resistance [114]. Inflammatory chemokine and cytokine production has been reported to be induced by several metabolic pathways implicated in the pathogenesis of DPN $[115,116 \cdot]$. Multiple studies have demonstrated higher systemic acute-phase proteins, cytokines, and chemokines in DPN [88•, 117•], recently reviewed by Bönhof et al. [118•]. Furthermore, rodent models of neuropathic pain associated with the metabolic syndrome and T2DM demonstrate elevated pro-inflammatory mediator expression in the serum [119] and the dorsal root ganglia $[119,120]$. The outcomes of studies examining the association of inflammatory biomarkers and painful-DPN have been variable. Numerous inflammatory markers have been associated with painful-DPN: C-reactive protein (CRP) [86], tumor necrosis factor- $\alpha$ (TNF- $\alpha$ ) [121], inducible nitric oxide synthase [121], and interleukin 6 [117•]. Additionally, inflammatory mediators have been shown to differentiate between painful- from painless-neuropathies of various etiologies, including elevated serum IL-2, TNF- $\alpha$, and reduced anti-inflammatory IL-10 [122]; IL-6 and IL-10 sural nerve biopsy expression [123]; and TNF- $\alpha$ in human Schwann cells [124].

\section{The Central Nervous System}

Technological advances in imaging modalities have enabled detailed in vivo investigation of the nervous system in DM. Key differences have been identified within the CNS in painful-DPN, using a variety of different techniques, especially advanced MR imaging modalities.

\section{Spinal Cord Changes in Painful-DPN}

We have identified a reduction in the cross-sectional area of the spinal cord in subjects with DPN in comparison to patient with DM without DPN, healthy controls, and disease control subjects with hereditary sensory motor neuropathy type 1A [125]. However, structural differences in the spinal cord area have not been found between subjects with painless- and painful-DPN [125]. Recent studies have indicated that spinal disinhibition, measured using the rate dependent depression (RDD) of the Hoffman reflex (H-reflex), may be a potential biomarker of spinally mediated pain to differentiate painfulfrom painless-DPN [126]. The RDD has been demonstrated to assess $\gamma$-aminobutyric acid (GABA) type A receptor-mediated spinal inhibitory function in neuropathic pain models of DM rats [127]. Impaired RDD was found in DM rat models of T1DM and T2DM with neuropathic pain phenotypes [62•]. Also, the RDD in groups of healthy controls and T1DM subjects with painful- and painless-DPN was evaluated and it was significantly impaired in those with painful-DPN. Patients with greater RDD attenuation had higher pain scores but no difference in measures of large or small fiber dysfunction, perhaps suggesting spinal inhibitory dysfunction may occur independent of PNS alterations in painful-DPN.

\section{Advanced MRI Studies of the Brain}

Functional MRI (fMRI) measures the activity of brain regions by detecting changes in the oxygenation of hemoglobin, the blood oxygen level dependent signal (BOLD). The neurological signature of physical pain has been identified by fMRI and includes activation of the venterolateral thalamus, dorsal posterior insula, and somatosensory cortex, as well as brain regions related to emotional pain processing, including the anterior insula and anterior cingulate cortex (ACC) [128-130].

\section{The Thalamus}

The thalamus receives somatosensory signals from the spinal cord where they are processed, modulated, and transmitted to higher brain centers. A variety of brain alterations have been demonstrated in DPN and painful-DPN using advanced imaging techniques that examine brain neurochemistry, microvascular blood flow, and functional changes. MR spectroscopy (MRS) enables the measurement of selected metabolites within the brain [131]. Our group has used MRS to show neuronal dysfunction within the thalamus, by reduced $\mathrm{N}$ acetyl aspartate (NAA) to choline ratio as a neuronal marker, in subjects with painless-DPN [132]. Furthermore, DM animal models of painful-DPN have shown that the thalamus may be responsible for central amplification of somatosensory signals $[133,134]$. Similarly, thalamic dysfunction appears to play a key role in human painful-DPN. We have recently shown preserved thalamic NAA and the GABA levels within the thalamus in patients with painful-DPN, whereas these levels were reduced in patients with painless-DPN [135, 136]. These findings suggest that neurochemical measures of the thalamic neuronal function and neurotransmitters may be essential for pain signal transmission and/or amplification in painful-DPN.

Recently, we performed a study administering exogenous perfusion contrast to subjects with painful- and painless-DSPN to compare the thalamic microvascular perfusion at rest [137•]. Subjects with DPN both demonstrated delayed bolus arrival time to the thalamus, but subjects with neuropathic pain had a significantly taller peak concentration, a higher mean cerebral blood 
volume, and the longest blood transit time compared to painless-DPN. Therefore, microvascular vasodilation within the thalamus may induce hyperperfusion which could be related to elevated thalamic neuronal activity. Finally, fMRI study of the brain has indicated there may be disruption in thalamocortical connectivity in painfulDPN. Cauda et al. measured resting state fMRI to determine temporal correlations of brain activity in a small number of subjects with painful-DPN and healthy control patients [138]. Compared with the control group, there was reduced synchrony between the somatosensory cortex and thalamic nuclei in painful-DPN patients.

\section{Descending Inhibition}

It is well recognized that the midbrain and medullary brain regions can exert bidirectional control over nociception [139]. The periaqueductal gray (PAG) and rostroventromedial medulla (RVM) are key sites for the control of descending pain modulation, disruption of which in rodent models of painful-DPN has been shown to lead to enhancement of descending pain facilitation $[140,141]$. In human studies, our group performed MRdynamic susceptibility contrast imaging at rest and under experimental pain, by applying heat pain to the lateral thigh where participants did not experience neuropathy [142]. During experimental pain, the time to peak concentration of contrast reduced in healthy volunteers but significantly increased in subjects with painful-DPN in the bilateral sensory cortices and thalami, perhaps indicating an underlying impairment in descending inhibition. Segerdahl et al. interrogated the ventrolateral PAG (vlPAG) using resting state fMRI and arterial spin labelling to determine cerebral blood flow at rest and during heat stimulation to the foot [143•]. The painfulDPN group demonstrated altered vlPAG functional connectivity, which correlated to their pain intensity and the cerebral blood flow changes induced by experimental thermal stimulation. These studies indicate that abnormalities within the descending pain modulatory system may result not only in reduced inhibition of pain but increased amplification of pain signals in painful-DPN.

\section{Higher Brain Centers}

The higher brain centers are involved in the localization of pain (e.g., somatosensory cortex) as well as the behavioral, cognitive, and emotional response to painful stimuli (e.g., ACC, amygdala, insular cortex). Using a technique known as voxel-based morphometry, we calculated the brain volumes in subjects with DPN and identified total brain volume reduction which was localized to the somatosensory regions [144, 145].
Furthermore, our group has performed the largest cohort study of brain volume changes in DPN and painfulDPN to date [146]. In painful-DPN, cortical atrophy is localized within the somatomotor cortex and insula. We have also demonstrated abnormal cortical interactions within the somatomotor network at rest which correlated with measures of pain and behavior in subjects with painful-DPN [147]. A recent study performed singlephoton emission computed tomography to assess cerebral blood flow (CBF) in 24 subjects with painful- and 20 painless-DPN [148]. The painful-DPN group demonstrated increased $\mathrm{CBF}$ within the right $\mathrm{ACC}$ and left nucleus accuumbens. However, the painless-DPN group demonstrated more severe neurophysiological neuropathic impairment which may be a potential confounding factor. Furthermore, application of thermal heat stimuli resulted in altered BOLD fMRI responses in painfulcompared with painless-DPN, seen in two studies [149, 150]. A pilot study within our group found greater BOLD response within the primary somatosensory cortex, lateral frontal, and cerebellar regions [149]. Whereas Tseng et al. demonstrated augmented responses in multiple limbic and striatal structures (i.e., ACC, superior frontal gyrus, medial thalamus, anterior insular cortex, lentiform nucleus, and premotor area) with the BOLD signal in the ACC and lentiform nucleus correlating with pain rating to thermal stimulation [150]. It is currently unknown whether the CNS changes described in these studies are a response to peripheral nervous system afferent inputs or a primary mechanism responsible for the maintenance of neuropathic pain.

\section{Conclusions}

Painful-DPN is a major cause of morbidity in patients with DM. Unfortunately, our understanding of why patients with DPN develop neuropathic pain remains inadequate (Fig. 1.). We have summarized the current evidence of the differences between painful- and painlessDPN (see Table 1.). However, there are limitations in many of the studies including small sample sizes, inappropriate definition of neuropathic pain and DPN, and measurement of multiple variables, leading to a risk of false positives. More recently, large, well-characterized cross-sectional cohort studies have given valid insights into the risk factors and somatosensory profiles of painful-DPN $[23 \bullet \bullet, 27 \bullet \bullet, 28 \bullet \cdot]$. Unfortunately, longitudinal studies which prospectively identify definitive differences in painful-DPN have not yet been performed, and would be logistically challenging and costly to perform. These limitations notwithstanding, painful-DPN seems to be associated with female gender, increased small 


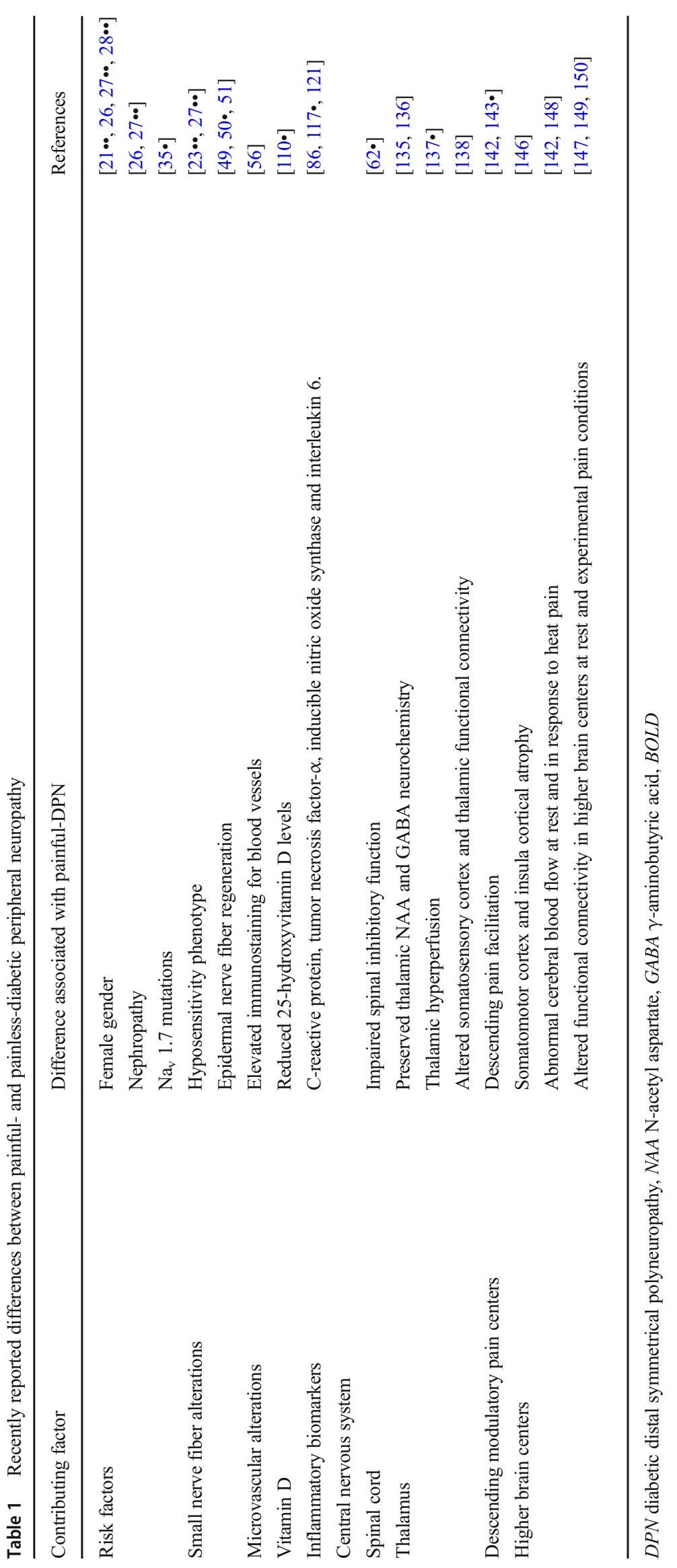


fiber injury and/or function, and peripheral/central vascular alterations. The role of autonomic dysfunction, vitamin D, inflammatory mediators, genetic factors, and methylglyoxal needs further clarification. Studies of the CNS demonstrate clear differences in painful- compared with painless-DPN. The spinal, somatomotor, limbic, thalamic, and ascending and descending modulatory systems demonstrate alterations using numerous testing techniques. However, what remains unclear is the causal relationship between painful-DPN and CNS changes. Further studies are necessary to determine whether these findings are the primary cause of neuropathic pain or adaptive to neuropathic afferent impulses. Irrespective of this, advanced MR imaging modalities have the potential for acting as biomarkers for monitoring therapeutic responses to treatments $[151 \bullet \bullet]$.

\section{Compliance with Ethical Standards}

Conflict of Interest Pallai Shillo, Gordon Sloan, Marni Greig, Leanne Hunt, Dinesh Selvarajah, Jackie Elliott, Rajiv Gandhi, and Iain D. Wilkinson declare that they have no conflict of interest.

Solomon Tesfaye reports grants from Impeto Medical; personal fees from Neurometrix, Pfizer, Miro, Worwag Pharma, Mundipharma, Merck, and Mitsubishi Pharma; and personal fees and other from Novo Nordisk.

Human and Animal Rights and Informed Consent This article does not contain any studies with human or animal subjects performed by any of the authors.

Open Access This article is distributed under the terms of the Creative Commons Attribution 4.0 International License (http:// creativecommons.org/licenses/by/4.0/), which permits unrestricted use, distribution, and reproduction in any medium, provided you give appropriate credit to the original author(s) and the source, provide a link to the Creative Commons license, and indicate if changes were made.

\section{References}

Papers of particular interest, published recently, have been highlighted as:

- Of importance

•. Of major importance

1. International Diabetes Federation. International diabetes federation: IDF Atlas. Brussels: Belgium; 2017. Available from: http:// diabetesatlas.org/resources/2017-atlas.html. Accessed 29 April 2019.

2. Dyck PJ, Kratz KM, Karnes JL, Litchy WJ, Klein R, Pach JM, et al. The prevalence by staged severity of various types of diabetic neuropathy, retinopathy, and nephropathy in a population-based cohort: the Rochester Diabetic Neuropathy Study. Neurology. 1993;43:817-24.

3. Pop-Busui R, Boulton AJM, Feldman EJ, Bril V, Freeman R, Malik RA, et al. Diabetic neuropathy: a position statement by the American Diabetes Association. Diabetes Care. 2017;40:
136-54 Recent position statement of Diabetic Neuropathy by the American Diabetes Association.

4. Tesfaye S, Chaturvedi N, Eaton SE, Ward JD, Manes C, IonescuTirgoviste $\mathrm{C}$, et al. Vascular risk factors and diabetic neuropathy. N Engl J Med. 2005;352:341-50 Prospective epidemiological study showing that, apart from glycemic control, incident neuropathy is associated with modifiable cardiovascular risk factors.

5. Tesfaye S, Boulton AJ, Dyck PJ, Freeman R, Horowitz M, Kempler P, et al. Diabetic neuropathies: update on definitions, diagnostic criteria, estimation of severity, and treatments. Diabetes Care. 2010;33:2285-93 Review by international panel of experts making recommendations in the clinical practice and research for DPN.

6. Sadosky A, Mardekian J, Parsons B, Hopps M, Bienen EJ, Markman J. Healthcare utilization and costs in diabetes relative to the clinical spectrum of painful diabetic peripheral neuropathy. J Diabetes Complicat. 2015;29:212-7.

7. Narres M, Kvitkina T, Claessen H, Droste S, Schuster B, Morbach $\mathrm{S}$, et al. Incidence of lower extremity amputations in diabetic compared with the non-diabetic population: a systematic review. PLoS One. 2017;12:e0182081. https://doi.org/10.1371/journal.pone. 0182081.

8. Daousi C, MacFarlane IA, Woodward A, Nurmikko TJ, Bundred PE, Benbow SJ. Chronic painful peripheral neuropathy in an urban community: a controlled comparison of people with and without diabetes. Diabet Med. 2004;21:976-82.

9. Van Acker K, Bouhassira D, De Bacquer D, Weiss S, Matthys K, Raemen $\mathrm{H}$, et al. Prevalence and impact on quality of life of peripheral neuropathy with or without neuropathic pain in type 1 and type 2 diabetic patients attending hospital outpatients clinics. Diabetes Metab. 2009;35:206-13.

10. Ziegler D, Rathmann W, Dickhaus T, Meisinger C, Mielck A, KORA Study Group. Neuropathic pain in diabetes, prediabetes and normal glucose tolerance: the MONICA/KORA Augsburg surveys S2 and S3. Pain Med. 2009;10:393-400.

11. Abbott CA, Malik RA, van Ross ER, Kulkarni J, Boulton AJ. Prevalence and characteristics of painful diabetic neuropathy in a large community-based diabetic population in the U.K. Diabetes Care. 2011;34:2220-4.

12. Alleman CJ, Westerhout KY, Hensen M, Chambers C, Stoker M, Long $\mathrm{S}$, et al. Humanistic and economic burden of painful diabetic peripheral neuropathy in Europe: a review of the literature. Diabetes Res Clin Pract. 2015;109:215-25 Review article of the epidemiology, humanistic burden and increasing healthcare costs associated with painful-DPN.

13. Binns-Hall O, Selvarajah D, Sanger D, Walker J, Scott A, Tesfaye S. One-stop microvascular screening service: an effective model for the early detection of diabetic peripheral neuropathy and the high-risk foot. Diabet Med. 2018;35:887-94.

14. Taylor-Stokes G, Pike J, Sadosky A, Chandran A, Toelle T. Association of patient-rated severity with other outcomes in patients with painful diabetic peripheral neuropathy. Diabetes Metab Syndr Obes. 2011;4:401-8.

15. Lauria G, Lombardi R. Small fiber neuropathy: is skin biopsy the holy grail? Curr Diab Rep. 2012;12:384-92.16.

16. Jensen TS, Backonja MM, Hernández Jiménez S, Tesfaye S, Valensi P, Ziegler D. New perspectives on the management of diabetic peripheral neuropathic pain. Diab Vasc Dis Res. 2006;3: 108-19.

17. Sloan G, Shillo P, Selvarajah D, Wu J, Wilkinson ID, Tracey I, et al. A new look at painful diabetic neuropathy. Diabetes Res Clin Pract. 2018;144:177-91.

18. Andersen ST, Witte DR, Dalsgaard EM, Andersen H, Nawroth P, Fleming T, et al. Risk factors for incident diabetic polyneuropathy in a cohort with screen-detected type 2 diabetes followed for 13 
years: ADDITION-Denmark. Diabetes Care. 2018;41:1068-75 Recent prospective study finding that cardiovascular risk factors are associated with incident DPN in T2DM.

19. Pop-Busui R, Lu J, Brooks MM, Albert S, Althouse AD, Escobedo J, et al. Impact of glycemic control strategies on the progression of diabetic peripheral neuropathy in the bypass angioplasty revascularization investigation 2 diabetes (BARI 2D) cohort. Diabetes Care. 2013;36:3208-15.

20. Callaghan BC, Gao LL, Li Y, Zhou X, Reynolds E, Banerjee M, et al. Diabetes and obesity are the main metabolic drives of peripheral neuropathy. Ann Clin Transl Neurol. 2018;5:397-40521.

21.• Hébert HL, Veluchamy A, Torrance N, Smith BH. Risk factors for neuropathic pain in diabetes mellitus. Pain. 2017;158:560-8 Recent review of the risk factors for painful-DPN.

22. Elliott J. Female sex: an independent risk factor for the development of painful neuropathy? Diabetes. 2006;55(Supp 1) Abstract, number 794-P

23.• Themistocleous AC, Ramirez JD, Shillo PR, Lees JG, Selvarajah $\mathrm{D}$, Orengo C, et al. The pain in neuropathy study (PiNS): a crosssectional observational study determining the somatosensory phenotype of painful and painless diabetic neuropathy. Pain. 2016;157:1132-45 Detailed cross-sectional study showing that the hyposensitivity sensory phenotype and increased severity of neuropathy are associated with painful-DPN.

24. Algeffari MA. Painful diabetic peripheral neuropathy among Saudi diabetic patients is common but under-recognized: multicenter cross-sectional study at primary health care setting. J Family Community Med. 2018;25:43-7.

25. Ziegler D, Landgraf R, Lobmann R, Reiners K, Rett K, Schnell O, et al. Painful and painless neuropathies are distinct and largely undiagnosed entities in subjects participating in an educational initiative (PROTECT study). Diabetes Res Clin Pract. 2018;139: 147-54.

26. Pai YW, Lin $\mathrm{CH}$, Lee IT, Chang MH, et al. Prevalence and biochemical risk factors of diabetic peripheral neuropathy with or without neuropathic pain in Taiwanese adults with type 2 diabetes mellitus. Diabetol Metab Syndr. 2018;12:11-6.

27.• Raputova J, Srotova I, Vlckova E, Sommer C, Üçeyler N, Birklein $\mathrm{F}$, et al. Sensory phenotype and risk factors for painful diabetic neuropathy: a cross-sectional observational study. Pain. 2017;158: 2340-53 Another detailed cross-sectional study finding painful-DPN is associated with severity of neuropathy and thermal hyposensitivity.

28.• Truini A, Spallone V, Morganti R, Tamburin S, Zenette G, Schenone A, et al. A cross-sectional study investigating frequency and features of definitely diagnosed diabetic painful polyneuropathy. Pain. 2018;159:2658-66 Large cross-sectional epidemiological study identifying female gender as the only risk factor associated with painful-DPN.

29. Abraham A, Barnett C, Katzberg HD, Lovblom LE, Perkins BA, Bril V. Sex differences in neuropathic pain intensity in diabetes. J Neurol Sci. 2018;388:103-6.

30. Sorge RE, Strath LJ. Sex differences in pain responses. Curr Opin Physiol. 2018;6:75-81.

31. Prabodha LBL, Sirisena ND, Dissanayake VHW. Susceptible and prognostic genetic factors associated with diabetic peripheral neuropathy: a comprehensive literature review. Int J Endocrinol. 2018;2018:8641942-9. https://doi.org/10.1155/2018/8641942.

32. Meng W, Deshmukh HA, Donnelly LA, Torrance N, Colhoun $\mathrm{HM}$, Palmer CN, et al. A genome-wide association study provides evidence of sex-specific involvement of Chr1p35.1 (ZSCAN20TLR12P) and Chr8p23.1 (HMGB1P46) with diabetic neuropathic pain. EBioMedicine. 2015;2:1386-93.

33. Meng W, Deshmukh HA, van Zuydam NR, Liu Y, Donnelly LA, Zhou K, et al. A genome-wide association study suggests an association of Chr8p21.3 (GFRA2) with diabetic neuropathic pain. Eur J Pain. 2015;19:392-9.

34. Li QS, Cheng P, Favis R, Wickenden A, Romano G, Wang H. SCN9A variants may be implicated in neuropathic pain associated with diabetic peripheral neuropathy and pain severity. Clin J Pain. 2015;31:976-82.

35. Blesneac I, Themistocleous AC, Fratter C, Conrad LJ, Ramirez JD, Cox JJ, et al. Rare Nav1.7 variants associated with painful diabetic peripheral neuropathy. Pain. 2017;159:469-80 Study showing that rare sodium channel genetic variants contribute to neuropathic pain in some patients with DPN.

36. Faber CG, Hoeijmakers JG, Ahn HS, Cheng X, Han C, Choi JS, et al. Gain of function Nav1.7 mutations in idiopathic small fiber neuropathy. Ann Neurol. 2012;71:26-39.

37. Galer BS, Gianas A, Jensen MP. Painful diabetic polyneuropathy: epidemiology, pain description, and quality of life. Diabetes Res Clin Pract. 2000;47:123-8.

38. Gore M, Brandenburg NA, Dukes E, Hoffman DL, Tai KS, Stacey B. Pain severity in diabetic peripheral neuropathy is associated with patient functioning, symptom levels of anxiety and depression, and sleep. J Pain Symptom Manag. 2005;30:374-85.

39. Veves A, Young MJ, Manes C, Boulton AJ. Differences in peripheral and autonomic nerve function measurements in painful and painless neuropathy. A clinical study. Diabetes Care. 1994;17: 1200-2.

40. Spallone V, Morganti R, D'Amato C, Cacciotti L, Fedele T, Maiello MR, et al. Clinical correlates of painful diabetic neuropathy and relationship of neuropathic pain with sensorimotor and autonomic nerve function. Eur J Pain. 2011;15:153-60.

41. Sorensen L, Molyneaux L, Yue DK. The level of small nerve fiber dysfunction dose not predict pain in diabetic neuropathy: a study using quantitative sensory testing. Clin J Pain. 2006;22:261-5.

42. Gandhi RA, Marques JL, Selvarajah D, Emery CJ, Tesfaye S. Painful diabetic neuropathy is associated with greater autonomic dysfunction than painless diabetic neuropathy. Diabetes Care. 2010;33:1585-90.

43. D'Amato C, Morganti R, Di Gennaro F, Marfia GA, Spallone V. A novel association between nondipping and painful diabetic polyneuropathy. Diabetes Care. 2014;37:2640-2.

44. Young RJ, Zhou TQ, Rodriguez E, Prescott RJ, Ewing DJ, Clarke BF. Variable relationship between peripheral somatic and autonomic neuropathy in patients with different syndromes of diabetic polyneuropathy. Diabetes. 1986;35:192-7.45.

45. Krämer HH, Rolke R, Bickel A, Birklein F. Thermal thresholds predict painfulness of diabetic neuropathies. Diabetes Care. 2004;27:2386-91.

46. Terkelsen AJ, Karlsson P, Lauria G, Freeman R, Finnerup NB, Jensen TS. The diagnostic challenge of small fibre neuropathy: clinical presentations, evaluations, and causes. Lancet Neurol. 2017;16:934-44.

47.• Smith SM, Dworkin RH, Turk DC, Baron R, Polydefkis M, Tracey I, et al. The potential role of sensory testing, skin biopsy, and functional brain imaging as biomarkers in chronic pain clinical trials: IMMPACT Considerations. J Pain. 2017;18:757-77 Review of the role of sensory testing, skin biopsy and brain imaging as diagnostic, prognostic, predictive and pharmacodynamic biomarkers in analgesic treatment trials.

48. Üçeyler N, Vollert J, Broll B, Riediger N, Langjahr M, Saffer N, et al. Sensory profiles and skin innervation of patients with painful and painless neuropathies. Pain. 2018;159:1867-76.

49. Cheng HT, Dauch JR, Porzio MT, Yanik BM, Hsieh W, Smith $A G$, et al. Increased axonal regeneration and swellings in intraepidermal nerve fibers characterize painful phenotypes of diabetic neuropathy. J Pain. 2013;14:941-7.

50. Bönhof GJ, Strom A, Püttgen S, Ringel B, Brüggemann J, Bódis $\mathrm{K}$, et al. Patterns of cutaneous nerve fibre loss and regeneration in 
type 2 diabetes with painful and painless polyneuropathy. Diabetologia. 2017;60:2495-503 Study demonstrating measures of cutaneous nerve fibre regeneration are enhanced in painful-DPN.

51. Galosi E, La Cesa S, Di Stefano G, Karlsson P, Fasolino A, Leone $\mathrm{C}$, et al. A pain in the skin. Regenerating nerve sprouts are distinctly associated with ongoing burning pain in patients with diabetes. Eur J Pain. 2018;22:1727-34.

52. Scheytt S, Riediger N, Braunsdorf S, Sommer C, Üçeyler N. Increased gene expression of growth associated protein-43 in skin of patients with early-stage peripheral neuropathies. J Neurol Sci. 2015:355:131-7.

53. Quattrini C, Tavakoli M, Jeziorska M, Kallinikos P, Tesfaye S, Finnigan J, et al. Surrogate markers of small fiber damage in human diabetic neuropathy. Diabetes. 2007;56:2148-54.

54. Cheung A, Podgomy P, Martinez JA, Chan C, Toth C. Epidermal axonal swellings in painful and painless diabetic peripheral neuropathy. Muscle Nerve. 2015;51:505-13.

55. Anand P, Terenghi G, Warner G, Kopelman P, Williams-Chestnut RE, Sinicropi DV. The role of endogenous nerve growth factor in human diabetic neuropathy. Nat Med. 1996;2:703-7.

56. Shillo P, Selvarajah D, Greig M, Wilkinson I, Yiangou Y, Donatien P, et al. Nerve and vascular biomarkers in skin biopsies differentiate painful from painless advanced diabetic peripheral neuropathy. Diabet Med. 2017;34(Supp 1):31-3.

57. Anand P, Bley K. Topical capsaicin for pain management: therapeutic potential and mechanism of action of the new high-concentration capsaicin 8\% patch. Br J Anaesth. 2011;107:490-502.

58. Obreja O, Rukwied R, Nagler L, Schmidt M, Schmelz M, Namer B. Nerve growth factor locally sensitizes nociceptors in human skin. Pain. 2018;159:416-26.

59. Alam U, Jeziorska M, Petropoulos IN, Asghar O, Fadavi H, Ponirakis G, et al. Diagnostic utility of corneal confocal microscopy and intra-epidermal nerve fibre density in diabetic neuropathy. PLoS One. 2017;12:e0180175. https://doi.org/10.1371/ journal.pone. 0180175 .

60. Malik RA, Kallinikos P, Abbott CA, van Schie CH, Morgan P, Efron N, et al. Corneal confocal microscopy: a non-invasive surrogate of nerve fibre damage and repair in diabetic patients. Diabetologia. 2003;46:683-8.

61. Jiang MS, Yuan Y, Gu ZX, Zhuang SL. Corneal confocal microscopy for assessment of diabetic peripheral neuropathy: a metaanalysis. Br J Opthalmol. 2016;100:9-14.

62. Marshall AG, Lee-Kubli C, Azmi S, Zhang M, Ferdousi M, Mixcoatl-Zecuatl T, et al. Spinal disinhibition in experimental and clinical painful diabetic neuropathy. Diabetes. 2017;66: 1380-90 Experimental and clinical study showing spinal inhibitory dysfunction may contribute to the pathogenesis of painful-DPN.

63. Kalteniece A, Ferdousi M, Petropoulos I, Azmi S, Adam S, Fadavi $\mathrm{H}$, et al. Greater corneal nerve loss at the inferior whorl is related to the presence of diabetic neuropathy and painful diabetic neuropathy. Sci Rep. 2018;8:3283. https://doi.org/10.1038/s41598-01821643-z.

64. Gasparotti R, Padua L, Briani C, Lauria G. New technologies for the assessment of neuropathies. Nat Rev Neurol. 2017;13:203-16.

65. Atherton DD, Facer P, Roberts KM, Misra VP, Chizh BA, Bountra $C$, et al. Use of the novel Contact Heat Evoked Potential Stimulator (CHEPS) for the assessment of small fibre neuropathy: correlations with skin flare responses and intra-epidermal nerve fibre counts. BMC Neurol. 2007;7:21. https://doi.org/10.1186/ 1471-2377-7-21.

66. Casanova-Molla J, Grau-Junyent JM, Morales M, Valls-Solé J. On the relationship between nociceptive evoked potentials and intraepidermal nerve fiber density in painful sensory polyneuropathies. Pain. 2011;152:410-8.
67. Chao CC, Tseng MT, Lin YJ, Yang WS, Hsieh SC, Lin YH, et al. Pathophysiology of neuropathic pain in type 2 diabetes: skin denervation and contact heat-evoked potentials. Hum Brain Mapp. 2013;34:2733-46.

68. Hansson P, Backonja M, Bouhassira D. Usefulness and limitations of quantitative sensory testing: clinical and research application in neuropathic pain states. Pain. 2007;129:256-9.

69. Cruccu G, Sommer C, Anand P, Attal N, Baron R, Garcia-Larrea $\mathrm{L}$, et al. EFNS guidelines on neuropathic pain assessment: revised 2009. Eur J Neurol. 2010;17:1010-8.

70. Tsigos C, White A, Young RJ. Discrimination between painful and painless diabetic neuropathy based on testing of large somatic nerve and sympathetic nerve function. Diabet Med. 1992;9:359-65.

71. Krishnan ST, Quattrini C, Jeziorska M, Malik RA, Rayman G. Abnormal LDIflare but normal quantitative sensory testing and dermal nerve fiber density in patients with painful diabetic neuropathy. Diabetes Care. 2009;32:451-5.

72. Rolke R, Magerl W, Campbell KA, Schalber C, Caspari S, Birklein F, et al. Quantitative sensory testing: a comprehensive protocol for clinical trials. Eur J Pain. 2006;10:77-88.

73.• Rolke R, Baron R, Maier C, Tölle TR, Treede RD, Beyer A, et al. Quantitative sensory testing in the German Research Network on Neuropathic Pain (DFNS): standardized protocol and reference values. Pain. 2006;123:231-43 QST protocol for the characterization the somatosensory phenotype of patients with neuropathic pain.

74. Umapathi T, Tan WL, Loke SC, Soon PC, Tavintharan S, Chan $\mathrm{YH}$. Intraepidermal nerve fiber density as a marker of early diabetic neuropathy. Muscle Nerve. 2007;35:591-8.

75. Løseth S, Stålberg E, Jorde R, Mellgren SI. Early diabetic neuropathy: thermal thresholds and intraepidermal nerve fibre density in patients with normal nerve conduction studies. J Neurol. 2008;255:1197-202.

76. Ragé M, Van Acker N, Knaapen MW, Timmers M, Streffer J, Hermans MP, et al. Asymptomatic small fiber neuropathy in diabetes mellitus: investigations with intraepidermal nerve fiber density, quantitative sensory testing and laser-evoked potentials. J Neurol. 2011;258:1852-64.

77. Cameron NE, Eaton SE, Cotter MA, Tesfaye S. Vascular factors and metabolic interactions in the pathogenesis of diabetic neuropathy. Diabetologia. 2001;44:1973-88.

78. Tesfaye S, Harris ND, Wilson RM, Ward JD. Exercise-induced conduction velocity increment: a marker of impaired peripheral nerve blood flow in diabetic neuropathy. Diabetologia. 1992;35: $155-9$.

79. Tesfaye S, Harris N, Jakubowski JJ, Mody C, Wilson RM, Rennie IG, et al. Impaired blood flow and arterio-venous shunting in human diabetic neuropathy: a novel technique of nerve photography and fluorescein angiography. Diabetologia. 1993;36:1266-74.

80. Tesfaye S, Malik R, Harris N, Jakubowski JJ, Mody C, Rennie IG, et al. Arterio-venous shunting and proliferating new vessels in acute painful neuropathy of rapid glycaemic control (insulin neuritis). Diabetologia. 1996;39:329-35.

81. Archer AG, Roberts VC, Watkins PJ. Blood flow patterns in painful diabetic neuropathy. Diabetologia. 1984;27:563-7.

82. Eaton SE, Harris ND, Ibrahim S, Patel KA, Selmi F, Radatz M, et al. Increased sural nerve epineurial blood flow in human subjects with painful diabetic neuropathy. Diabetologia. 2003;46: 934-9.

83. Tsigos C, Reed P, Weinkove C, White A, Young RJ. Plasma norepinephrine in sensory diabetic polyneuropathy. Diabetes Care. 1993;16:722-7.

84. Tack CJ, van Gurp PJ, Holmes C, Goldstein DS. Local sympathetic denervation in painful diabetic neuropathy. Diabetes. 2002;51:3545-53. 
85. Quattrini C, Harris ND, Malik RA, Tesfaye S. Impaired skin microvascular reactivity in painful diabetic neuropathy. Diabetes Care. 2007;30:655-9.

86. Doupis J, Lyons TE, Wu S, Gnardellis C, Dinh T, Veves A. Microvascular reactivity and inflammatory cytokines in painful and painless peripheral diabetic neuropathy. J Clin Endocrinol Metab. 2009;94:2157-63.

87. Vas PR, Sharma S, Rayman G. Distal sensorimotor neuropathy: improvements in diagnosis. Rev Diabet Stud. 2015;12:29-47.

88. Herder C, Kannenberg JM, Huth C, Carstensen-Kirbgerg M, Rathmann W, Koening W, et al. Proinflammatory cytokines predict the incidence and progression of distal sensorimotor polyneuropathy: KORA F4/FF4 Study. Diabetes Care. 2017;40: 569-76 Prospective study finding IL-6 and TNF $-\alpha$ are associated with incident DPN.

89. Deguchi T, Hashiguchi T, Horinouchi S, Uto T, Oku H, Kimura K, et al. Serum VEGF increases in diabetic polyneuropathy, particularly in the neurologically active symptomatic stage. Diabet Med. 2009;26:247-52.

90. Quattrini C, Jeziorska M, Boulton AJ, Malik RA. Reduced vascular endothelial growth factor expression and intra-epidermal nerve fiber loss in human diabetic neuropathy. Diabetes Care. 2008;31: $140-5$.

91. Yuen KC, Baker NR, Rayman G. Treatment of chronic painful diabetic neuropathy with isosorbide dinitrate spray: a double-blind placebo-controlled cross-over study. Diabetes Care. 2002;25: 1699-703.

92. Rayman G, Baker NR, Krishnan ST. Glyceryl trinitrate patches as an alternative to isosorbide dinitrate spray in the treatment of chronic painful diabetic neuropathy. Diabetes Care. 2003;26: $2697-8$

93. Obrosova IG. Diabetic painful and insensate neuropathy: pathogenesis and potential treatments. Neurotherapeutics. 2009;6(4): $638-47$

94. Dewanjee S, Das S, Das AK, Bhattacharjee N, Dihingia A, Dua TK. Molecular mechanism of diabetic neuropathy and its pharmacotherapeutic targets. Eur J Pharmacol. 2018;833:472-523.

95. Shamsaldeen YA, Mackenzie LS, Lione LA, Benham CD. Methylglyoxal, a metabolite increased in diabetes is associated with insulin resistance, vascular dysfunction and neuropathies. Curr Drug Metab. 2016;17:359-67.

96. Huang Q, Chen Y, Gong N, Wang YX. Methylglyoxal mediates streptozotocin-induced diabetic neuropathic pain via activation of the peripheral TRPA1 and Nav1.8 channels. Metabolism. 2016;65:463-74.

97. Bierhaus A, Fleming T, Stoyanov S, Leffler A, Babes A, Neacsu $\mathrm{C}$, et al. Methylglyoxal modification of Nav1.8 facilitates nociceptive neuron firing and causes hyperalgesia in diabetic neuropathy. Nat Med. 2012;18:926-33.

98. Hansen CS, Jensen TM, Jensen JS, Nawroth P, Fleming T, Witte $\mathrm{DR}$, et al. The role of serum methylglyoxal on diabetic peripheral and cardiovascular autonomic neuropathy: the ADDITION Denmark study. Diabetes Metab. 2015;32:778-85.

99. Zhou J, Zhou S. Inflammation: therapeutic targets for diabetic neuropathy. Mol Neurobiol. 2014:49:536-46.

100. Cakici N, Fakkel TM, van Neck JW, Verhagen AP, Coert JH. Systematic review of treatments for diabetic peripheral neuropathy. Diabet Med. 2016;33:1466-76.

101. Holick MF, Vitamin D. Deficiency. N Engl J Med. 2007;357:266-81.

102. Powanda MC. Is there a role for vitamin D in the treatment of chronic pain? Inflammopharmacology. 2014;22:327-32.

103. Danescu LG, Levy S, Levy J. Vitamin D and diabetes mellitus. Endocrine. 2009;35:11-7.

104. Mathieu C. Vitamin D and diabetes: where do we stand? Diabetes Res Clin Pract. 2015;108:201-9.
105. Fukuoka M, Sakurai K, Ohta T, Kiyoki M, Katayama I. Tacalcitol, an active vitamin D3, induces nerve growth factor production in human epidermal keratinocytes. Skin Pharmacol Appl Ski Physiol. 2001;14:226-33.

106. Riaz S, Malcangio M, Miller M, Tomlinson DR. A vitamin D(3) derivative (CB1093) induces nerve growth factor and prevents neurotrophic deficits in streptozotocin-diabetic rats. Diabetologia. 1999;42:1308-13.

107. Tague SE, Smith PG. Vitamin D receptor and enzyme expression in dorsal root ganglia of adult female rats: modulation by ovarian hormones. J Chem Neuroanat. 2011:41:1-12.

108. Tague SE, Clarke GL, Winter MK, McCarson KE, Wright DE, Smith PG. Vitamin D deficiency promotes skeletal muscle hypersensitivity and sensory hyperinnervation. J Neurosci. 2011;31: 13728-38.

109. Esteghamati A, Fotouhi A, Faghihi-Kashani S, Hafezi-Nejad N, Heidari B, Sheikhbahaei S, et al. Non-linear contribution of serum vitamin D to symptomatic diabetic neuropathy: a case-control study. Diabetes Res Clin Pract. 2016;111:44-50.

110. Shillo P, Selvarajah D, Greig M, Gandhi R, Rao G, Wilkinson ID, et al. Reduced vitamin D levels in painful diabetic peripheral neuropathy. Diabet Med. 2018;36:44-51. https://doi.org/10.1111/ dme.13798. Cross-sectional study demonstrating that reduced serum 25-hydroxyvitamin $D$ levels are associated with painful-DPN.

111. Lee $P$, Chen R. Vitamin D as an analgesic for patients with type 2 diabetes and neuropathic pain. Arch Intern Med. 2008;168:771-2.

112. Shehab D, Al-Jarallah K, Abdella N, Mojiminiyi OA, Al Mohamedy H. Prospective evaluation of the effect of short-term oral vitamin $\mathrm{d}$ supplementation on peripheral neuropathy in type 2 diabetes mellitus. Med Princ Pract. 2015;24:250-6.

113. Basit A, Basit KA, Fawwad A, Shaheen F, Fatima N, Petropoulos IN, et al. Vitamin D for the treatment of painful diabetic neuropathy. BMJ Open Diabetes Res Care. 2016;4:e00148. https://doi. org/10.1136/bmjdrc-2015-000148.

114. Pop-Busui R, Ang L, Holmes C, Gallgher K, Feldman E. Inflammation as a therapeutic target for diabetic neuropathies. Curr Diab Rep. 2016;16(3):29. https://doi.org/10.1007/s11892016-0727-5.

115. Jin HY, Park TS. Role of inflammatory biomarkers in diabetic peripheral neuropathy. J Diabetes Investig. 2018;9:1016-8.

116. Feldman EL, Nave KA, Jensen TS, Bennett DL. New horizons in diabetic neuropathy: mechanisms, bioenergetics, and pain. Neuron. 2017;93:1296-313 Review of the recent discoveries into the pathogenesis of DPN and painful-DPN.

117. Herder C, Bongaerts BW, Rathmann W, Heier M, Kowall B, Koenig W, et al. Differential association between biomarkers of subclinical inflammation and painful polyneuropathy: results from the KORA F4 study. Diabetes Care. 2015;38:91-6 Study showing that biomarkers of subclinical and vascular inflammation are associated with painful-DPN.

118. Bönhof GJ, Herder C, Strom A, Papanas N, Roden M, Ziegler D. Emerging biomarkers, tools, and treatments for diabetic polyneuropathy. Endocr Rev. 2018;40:153-92. https://doi.org/10. 1210/er.2018-00107. Detailed review of the biomarkers and treatments for DPN.

119. Zhang C, Ward J, Dauch JR, Tanzi RE, Cheng HT. Cytokinemediated inflammation mediates painful neuropathy from metabolic syndrome. PLoS One. 2018;13:e0192333. https://doi.org/10. 1371/journal.pone.0192333.

120. Cheng HT, Dauch JR, Oh SS, Hayes JM, Hong Y, Feldman EL. p38 mediates mechanical allodynia in a mouse model of type 2 diabetes. Mol Pain. 2010;6:28. https://doi.org/10.1186/17448069-6-28. 
121. Purwata TE. High TNF-alpha plasma levels and macrophages iNOS and TNF-alpha expression as risk factors for painful diabetic neuropathy. J Pain Res. 2011;4:169-75.

122. Uçeyler N, Rogausch JP, Toyka KV, Sommer C. Differential expression of cytokines in painful and painless neuropathies. Neurology. 2007;69:42-9.

123. Üçeyler N, Riediger N, Kafke W, Sommer C. Differential gene expression of cytokines and neurotrophic factors in nerve and skin of patients with peripheral neuropathies. J Neurol. 2015;262:203-12.

124. Empl M, Renaud S, Erne B, Fuhr P, Straube A, Schaeren-Wiemers $\mathrm{N}$, et al. TNF-alpha expression in painful and nonpainful neuropathies. Neurology. 2001;56:1371-7.

125. Selvarajah D, Wilkinson ID, Emery CJ, Harris ND, Shaw PJ, Witte DR, et al. Early involvement of the spinal cord in diabetic peripheral neuropathy. Diabetes Care. 2006;29:2664-9.

126. Lee-Kubli C, Marshall AG, Malik RA, Calcutt NA. The H-reflex as a biomarker for spinal disinhibition in painful diabetic neuropathy. Curr Diab Rep. 2018;18:1. https://doi.org/10.1007/s11892018-0969-5.

127. Lee-Kubli CA, Calcutt NA. Altered rate-dependent depression of the spinal H-reflex as an indicator of spinal disinhibition in models of neuropathic pain. Pain. 2014;155:250-60.

128. Fomberstein K, Qadri S, Ramani R. Functional MRI and pain. Curr Opin Anaesthesiol. 2013;26:588-93.

129. Tracey I. Imaging pain. Br J Anaesth. 2008;101:32-9.

130. Wager T, Atlas L, Lindquist M, Roy M, Woo C, Kross E. An fMRI-based neurologic signature of physical pain. N Engl J Med. 2013;368:1388-97.

131. Buonocore MH, Maddock RJ. Magnetic resonance spectroscopy of the brain: a review of physical principles and technical methods. Rev Neurosci. 2015;26:609-32.

132. Selvarajah D, Wilkinson ID, Emery CJ, Shaw PJ, Griffiths PD, Gandhi R, et al. Thalamic neuronal dysfunction and chronic sensorimotor distal symmetrical polyneuropathy in patients with type 1 diabetes mellitus. Diabetologia. 2008;51:2088-92.

133. Fischer TZ, Tan AM, Waxman SG. Thalamic neuron hyperexcitability and enlarged receptive fields in the STZ model of diabetic pain. Brain Res. 2009;1268:154-61.

134. Freeman OJ, Evans MH, Cooper GJ, Petersen RS, Gardiner NJ. Thalamic amplification of sensory input in experimental diabetes. Eur J Neurosci. 2016;44:1779-86.

135. Gandhi R, Selvarajah D, Wilkinson I, Emery C, Shaw PJ, Griffiths $\mathrm{P}$, et al. Preservation of thalamic neuronal function may be a prerequisite for pain perception in diabetic neuropathy. Diabetologia. 2006;49(Supp 1):2088-92.

136. Shillo P, Selvarajah D, Greig M, Rao D, Edden R, Wilkinson I, et al. Painless diabetic peripheral neuropathy is characterised by reduced thalamic gamma-aminobutyric acid (GABA). Diabet Med. 2016;33(Supp 1):162.

137. Selvarajah D, Wilkinson ID, Gandhi R, Griffiths PD, Tesfaye S. Microvascular perfusion abnormalities of the thalamus in painful but not painless diabetic polyneuropathy: a clue to the pathogenesis of pain in type 1 diabetes. Diabetes Care. 2011;34:718-20 Study demonstrating increased microvascular perfusion within the thalamus is associated with painful-DPN.

138. Cauda F, Sacco K, D'Agata F, Duca S, Cocito D, Geminiani G, et al. Low-frequency BOLD fluctuations demonstrate altered thalamocortical connectivity in diabetic neuropathic pain. BMC Neurosci. 2009;10:138. https://doi.org/10.1186/1471-2202-10-138.

139. Ossipov MH, Morimura K, Porreca F. Descending pain modulation and chronification of pain. Curr Opin Support Palliat Care. 2014;8:143-51.

140. Silva M, Amorim D, Almeida A, Tavares I, Pinto-Ribeiro F, Morgado C. Pronociceptive changes in the activity of rostroventromedial medulla (RVM) pain modulatory cells in the streptozotocin-diabetic rat. Brain Res Bull. 2013;96:39-44.

141. Morgado C, Terra PP, Tavares I. Neuronal hyperactivity at the spinal cord and periaqueductal grey during painful diabetic neuropathy: effects of gabapentin. Eur J Pain. 2010;14:693-9.

142. Greig M, Wilkinson ID, Shillo P, Selvarajah D, Gandhi R, Tesfaye S. Impaired hemodynamic response to thermal pain in patients with painful diabetic neuropathy. Diabetes. 2018;67(Supp 1): 568-P. https://doi.org/10.2337/db18-568-P.

143. Segerdahl AR, Themistocleous AC, Fido D, Bennett DL, Tracey I. A brain-based pain facilitation mechanism contributes to painful diabetic polyneuropathy. Brain. 2018;141:357-64 Study finding vIPAG altered function may contribute to neuropathic pain in painful-DPN.

144. Ashburner J, Friston KJ. Voxel-based morphometry-the methods. Neuroimage. 2000;11:805-21.

145. Selvarajah D, Wilkinson ID, Maxwell M, Davies J, Sankar A, Boland E, et al. Magnetic resonance neuroimaging study of brain structural differences in diabetic peripheral neuropathy. Diabetes Care. 2014;37:1681-8.

146. Selvarajah D, Heiberg-Gibbons F, Wilkinson ID, Gandhi R, Tesfaye S. A magnetic resonance imaging volumetry study of regional brain atrophy in diabetic peripheral neuropathy. Diabetes. 2018;67(Supp 1):550-P. https://doi.org/10.2337/db18550-P.

147. Selvarajah D, Awadh M, Gandhi R, Wilkinson ID, Tesfaye S. Alterations in somatomotor network functional connectivity in painful diabetic neuropathy - a resting state functional magnetic resonance imaging study. Diabetes. 2018;67(Supp 1):61-OR. https://doi.org/10.2337/db18-61-OR.

148. Watanabe K, Hirano S, Kojima K, Nagashima K, Mukai H, Sato $\mathrm{T}$, et al. Altered cerebral blood flow in the anterior cingulate cortex is associated with neuropathic pain. J Neurol Neurosurg Psychiatry. 2018;89:1082-7.

149. Selvarajah D, Gandhi R, Hunter M, Emery C, Griffiths P, Tesfaye $\mathrm{S}$, et al. A functional magnetic resonance imaging study demonstrating alterations in brain responses to acute pain stimulation in diabetic neuropathy. Diabetologia. 2007;50(Supp 1):448-S9.

150. Tseng MT, Chiang MC, Chao CC, Tseng WY, Hsieh ST. fMRI evidence of degeneration-induced neuropathic pain in diabetes: enhanced limbic and striatal activations. Hum Brain Mapp. 2013;34:2733-46.

151.• Tesfaye S, Selvarajah D, Gandhi R, Greig M, Shillo P, Fang F, et al. Diabetic peripheral neuropathy may not be as its name suggests: evidence from magnetic resonance imaging. Pain. 2016;157(Supp 1):S72-80 Review of the MRI studies into painful-DPN.

Publisher's Note Springer Nature remains neutral with regard to jurisdictional claims in published maps and institutional affiliations. 